\title{
Simulating Population Dynamics with an Agent Based and Microsimulation Based Framework
}

\author{
Karandeep Singh ${ }^{1}$, Mazhar Sajjad ${ }^{1}$, Chang-Won Ahn ${ }^{2}$ \\ ${ }^{1}$ Department of Computer \& SW, University of Science Technology, Daejeon, South Korea \\ ${ }^{2}$ Department of BigData SW Research, Electronics \& Telecommunications Research Institute, Daejeon, South Korea
}

\begin{abstract}
Simulation has been an important way of modeling and for predicting and analyzing population dynamics related phenomena. There have been many advances in this field and various techniques have been applied for prediction and understanding. Microsimulation and multi agent based simulation are two of the most suitable techniques which give us results that could be related to the real world phenomena. Applying these techniques enable us to perform various multi-dimensional policy experiments on demography related processes. Of these two techniques, microsimulation has been around for longer time, but of late, agent based techniques have become very popular. These models are being used more and more in the social sciences. Both of techniques have similarities and as well as differences. We will explore these techniques and propose a new framework which has positive aspects of both the approaches.
\end{abstract}

Index Terms - Agent Based Modeling, Microsimulation, Demography, Social Simulation, Population Dynamics.

\section{Introduction}

Population dynamics deals with changes in the size and age composition of populations, and the various concerned factors concerning those changes. It deals with the way populations are affected by factors such as mortality, health, education, economy, matrimony, fertility, migration, welfare etc. Many countries of the world have undergone decline in the population growth. Majority of the OECD countries have experienced reduction in fertility levels, with in some cases, the fertility rate dipping below the required rate for population sustainability. The recent studies have shown that this decline is temporary and will reverse itself [1], [2], [3]. However, countries like Korea have not experienced this turnaround and low fertility rate continues to be a major concern for the government. The population of Korea is declining with low fertility rate. The 2014 fertility rate stood at 1.21 children per woman, well below sustainable level of 2.08 [4], [5].

To avert off future problems, particularly related with the economy, which will arise if population continues to decline like this, policy makers have begun making changes to incentivize and advertise larger family sizes. But the results of these efforts have not been satisfactory. In spite of the fact that government is spending large munts of money on various public policies regarding the public welfare, the output of that spending is debatable. Scientist have been trying various techniques for this purpose and have been trying to optimize the government spending and optimize the results from the spending.

Simulation and modelling are a good way to solve this problem. These techniques have been used to analyse the behaviour of the population, people and predict the possible outcome of the policy application. Scientist can create virtual environments and virtual societies to experiment upon. Experimenting on real population is long, expensive and an unethical process.

The factors affecting the population dynamics could be classified as micro, meso and macro level. Micro level focusses on individual decision making processes. E.g. what factors affect decisions regarding timing and no. of children a couple would have. Meso level approaches consider individuals as social actors who take their decisions while interacting with people in their relations. E.g. how place of residence could affect no. of children couple would have. Macro level approaches considers effect of cultural and institutional setting on individuals.

Popular approaches for simulation are statistical, microsimulation or agent based models (ABM). Statistical approaches are too general and don't help to solve problems like analysing or prediction. The other two are very useful for obtaining and understanding micro-macro links and outcomes. We shall discuss microsimulation and agent based modelling approaches. The purpose of this paper is to introduce microsimulation and agent based simulation in simplest of terms and clear understanding of differences in these two. It also analyzes the drawbacks of these techniques in context of demographic simulations and population dynamics simulations and how we can overcome these. The rest of the paper is organized as follows: Section 2 will cover Microsimulation, Section 3 will discuss Agent Based Modeling and Section 4 discuses about comparison, drawbacks and improvement suggestion to these simulation techniques, section 5 is conclusion.

\section{Microsimulation}

\section{A. Microsimulation Process}

The microsimulation as a technique for socio-economic modelling was pioneered by Orcutt (1957) [6], who identified and represented individual actors in the economic system through a way in which their behavior changed temporally. The early adoption of this technique for socio-economic modelling was hindered by the limited computer resources and lack of data about temporal behavior.

Microsimulation is based on a large sample of population (e.g. individuals, households, firms, etc.), treated as a unit. The basic concept is simply to represent the elementary units with unique characteristics rather than using aggregate counts such as output areas from the census. Often, this is achieved by generating a synthetic population to represent imaginary rather 
than real people using aggregate data. In this manner, particular characteristics are associated with a particular unit or individual, so that the distributions of the simulated/synthetic population match the real or modelled distributions. A simple representation of microsimulation concept can be visualized in Figure 1.

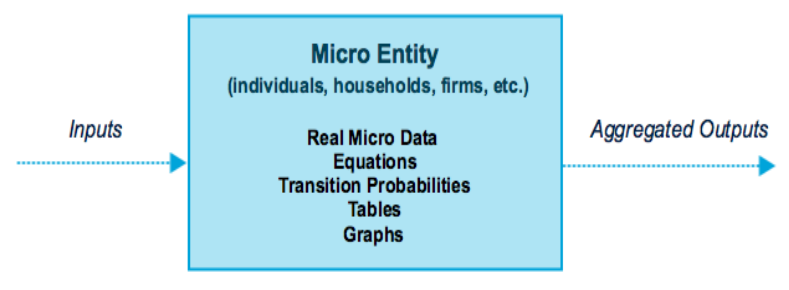

Fig. 1 Microsimulation Concept

Once the population has been created in this way, the model is used for some purpose such as policy analysis. Various transition rates drive the simulation and determine the chances that these units will undergo any particular changes as they "age". Microsimulation is not intended as an explanatory tool, but as a means of predicting the future behavior. It basically studies and predicts the evolution of a population using a model for individuals. It tries to study in depth the macro consequences of the micro behavior. Aggregate statistics can be calculated by "adding" up individual outputs and can be used as estimates of the future characteristics of the population of the units.

Microsimulation does not attempt to model interaction between units, nor attempt to contain the motivations or intentions of the units. There is no interaction between the units of population. A random philosophical mechanism process (signifies link to stochastic processes) takes care of the unit "aging" task. There is no feedback from the macro to the micro level. The process of creating individuals based on aggregate data addresses the problem of assigning average group attributes to individual entities, and assuming an ecological correlation between them. Microsimulation are developed with validation ambitions by focusing on the aggregate information of groups of individuals to predict policy impacts [7].

Types of micro-simulation can be defined from a number of perspectives, viz. from types of population, type of temporal variation, case vs. time based etc. Broadly they could be classified as:

1) Mathematical vs. Behavioral: mathematical models are used for estimating distribution and budget changes while individual behavioral reaction are ignored. In contrast, behavioral model has changes in behavior according to changes in policies.

2) Static vs. Dynamic: static model perform analysis for a time point or several pints in time whereas dynamic models update attributes that are defined for each time step with sort of probabilistic algorithm.

\section{B. Demographic Microsimulation Models.}

There have been a lot of work done in the field of microsimulation. Micro-simulation has developed mainly in the advanced countries like USA, Canada, UK, Sweden, France, and Australia since 1990's. Some of the famous examples of models are DYNASIM3 (US), DYNACAN (Canada), MOSART (Norway), SESIM (Sweden), SAGE (UK), DYNAMOD (Australia), APPSIM (Australia), DESTINIE (France), and SVERIGE (Sweden). All these models have similar structure: initial population, simulation cycle and population outputs. Within each cycle a set of functions calculates the probabilities.

\section{Agent Based Simulation}

\section{A. Agent Based Simulation Process}

Agent Based Models (ABM) can be traced back to Von Neumann [8]. Well-known pioneering examples of ABMs are John Conway's Game of Life [9] and the famous segregation model by Schelling [10]. ABMs basically simulate interactions of autonomous agents in order to study the emergence of global features from the bottom up, the main idea is that interaction can produce the behavior which is more/less than the aggregated sum of the individualistic activities.

Each agent has set of rules through which it interacts with the others agents and the simulated environment. Agents can access information (sometimes in light of past experiences) and make new decisions (signifies link to artificial intelligence). Agents have properties like autonomy, heterogeneity, pro-active, reactive, perceptive, bounded rationality, mobility, learning, interactive etc. [11], [12], [13], [14], [15] and many others. The agent based social simulation for demography was pioneered by Billari and Prskawetz [16]. The concept of agent working could simply be stated as shown in Figure 2.

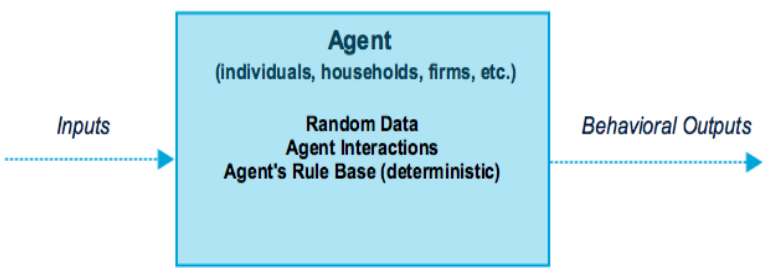

Fig. 2 Agent Based Simulation Concept

ABMs agents dynamically adapt and react to the everchanging simulation environment. ABMs' base is a behavioral modelling and they can easily take feedback effects between micro and macro levels, and for this reason they appear to the ideal complement to the microsimulation models.

\section{B. Agent Based Demographic Models}

There have been a variety of models in agent based simulation. But one of the property of the agent based simulation models is that they are specific to the problem. There has never been a generalization of the agent based model. Variety of models have been proposed in demographic context [24], some example are: family formation: Todd, Billari \& Simao [17]; Billari [18]; Billari et al. [19], [20]; Hills and Todd [21]; Aparicio-Diaz et al. [22]; E. Frydenlund [23], Migration: Heiland [25]; Kniveton, Smith \& Wood [26]; 
Willkens [27], Residential Mobility: Benenson, Orner \& Hatna [28], Household Dynamics: Geard et al. [29]. As we can see that these models are specific in nature and the high-level framework which brings together various aspects of the agent based simulation is missing.

\section{Comparing and Combining Both the Approaches}

\section{A. Differences and Drawbacks of Mirosimulation and Agent Based Modelling.}

We have seen till now that many microsimulation agent based approaches have been in design to model and simulate the demographic processes. But there are variety of drawbacks related with these two approaches. The aim of the simulation should be the predicting and analyzing the transition link from micro to the macro. This concept can be viewed as in Figure 3.

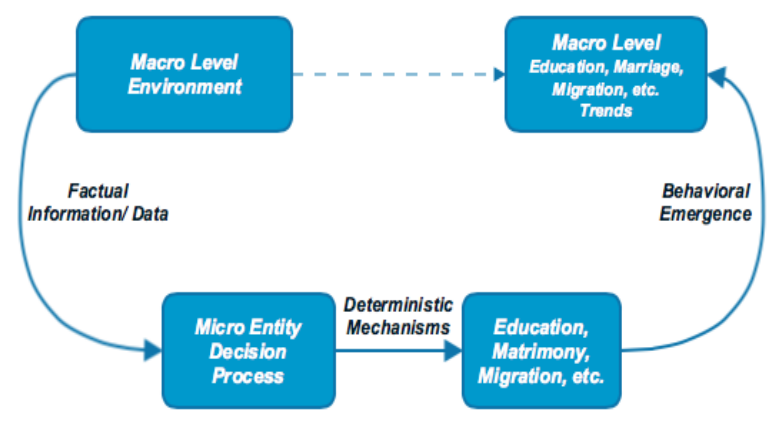

Fig. 3 Micro - Macro Link

The basic issue with microsimulation is interaction of agents. In these kind of models, agents don't interact, the simulation consists of repeatedly changing simulated units according to the transition probabilities an adding up the output. The aggregated output would be outlier if agents do interact among themselves. The purpose of the approach is thus, validation, not emergence [30]. This can't be related to reality, especially when we dealing with the demographic and population dynamics processes.

In case of $\mathrm{ABM}$, the initial conditions (individualistic and environmental) are not attempted to be like that of real world. The values are generally taken from the uniform random distributions. This initial choice can affect the output. E.g., emergence of trends from simulation that we can hardly relate to the real world [31]. The requirement to specify all the rules means it is difficult to apply for real world problems.

Table 1 summarizes the differences between two approaches [32]. Important to note here is probabilistic and deterministic rules to move to next 'state'. Probabilistic means certain probability that it would move to next state, whereas deterministic means certain rule set would be used to arrive to next state. Only if those conditions are satisfied, the transition to the next state would take place. Also worth noting is output form: aggregated output in microsimulation and behavioral emergence after the agent interaction in agent based simulation techniques.
TABLE I Comparison between Microsimulation and Agent Based Simulation

\begin{tabular}{|c|c|c|}
\hline Characteristic & Microsimulation & Agent Based \\
\hline Origin & Stochastic Process & Artificial Intelligence \\
\hline Purpose & Projection/Validation & Explanation/Emergence \\
\hline $\begin{array}{l}\text { Research } \\
\text { Approach }\end{array}$ & $\begin{array}{l}\text { Deduction; } \\
\text { aggregating individual } \\
\text { units }\end{array}$ & $\begin{array}{l}\text { Induction ; behavioural } \\
\text { emergence of units }\end{array}$ \\
\hline Analysis Method & $\begin{array}{l}\text { Infer from aggregate } \\
\text { to individual }\end{array}$ & $\begin{array}{c}\text { Infer from individual to } \\
\text { aggregate }\end{array}$ \\
\hline Applications & $\begin{array}{l}\text { Econometrics, traffic } \\
\text { modelling }\end{array}$ & Social simulation \\
\hline Basic Constituents & $\begin{array}{l}\text { Micro units and } \\
\text { aggregate entities }\end{array}$ & $\begin{array}{l}\text { Individual agents } \\
\text { connected with each } \\
\text { other }\end{array}$ \\
\hline $\begin{array}{c}\text { Possible } \\
\text { interaction }\end{array}$ & 2 & $2+$ \\
\hline $\begin{array}{l}\text { Constituent unit } \\
\text { decision process }\end{array}$ & Probabilistic & Deterministic \\
\hline Number of agents & Higher & Fewer \\
\hline $\begin{array}{c}\text { Complexity of } \\
\text { agents }\end{array}$ & Lower & Higher \\
\hline $\begin{array}{l}\text { Communication } \\
\text { between agents }\end{array}$ & No & Yes \\
\hline $\begin{array}{l}\text { Development of } \\
\text { Expected output }\end{array}$ & $\begin{array}{l}\text { Built through } \\
\text { transition probabilities }\end{array}$ & $\begin{array}{c}\text { Built thorough agent's } \\
\text { rule set }\end{array}$ \\
\hline Agents Properties & $\begin{array}{c}\text { Units with provided } \\
\text { data and transition } \\
\text { rules }\end{array}$ & Intelligent agents \\
\hline
\end{tabular}

\section{B. Feeding Microsimulation Data to Agent Based Modelling}

Now a days, emphasis is being laid to add behaviour to the microsimulation and add data to the agent based models. Most of the agent based simulation are started with the random distributions of the data and initial conditions to start the simulation. Similarly random values are also used for spatial distribution of the agents to determine the unmeasured aspects of the agents, and to discover the exogenous factors. An alternative approach to this can be using the principles of the microsimulation and initializing the simulation with that data. E.g. surveys for initial conditions, derived regression equations for evolution of the variables, and empirical distributions for calculation of the new values.

The choice of the initial conditions can affect the output of the simulation considerably and random initialization of the variables would just produce the erroneous outputs of the simulation. If we are using agent based simulation and initial data from the census, the model would become like agents based microsimulation with all the transition probabilities equal to 1. A hybrid model would contain some deterministic transition rules to generate behaviour like that of agent based model and some probabilistic rules where there is lack of information like in a microsimulation model.

Several efforts have been made for the introduction the empirical data into ABMs. [22], [23], [33], [34], [35]. These examples show how random initialization can be addressed successfully by feeding the model with real data. It is know 
that the random initialization of data is particularly inadequate in the distribution of the links between agents and to form a social circle or a network [36]. One popular example of the improvement of the ABM model output by introducing data is Eurovision song contest [37].

Feeding the model with the actual data could be particularly helpful in demographic simulation as quantitative \& qualitative data is now often collected regularly for demography changes. Some aspects of microsimulation, such as basing the simulation on survey samples and using probability transition methods to determine changes in the values of agent parameters, can be usefully applied to the design of ABM.

Agent-based models usually follow deterministic rules which are event based rather than using transition probabilities. However, the limitations of modelling or the lack of required data frequently make it difficult to implement explicit rules and there is no way out but to other solutions, one of which is to use transition probabilities [38].

Therefore we can move towards the direction of rearranging the modelling steps:

1) Design the agent based model.

2) Initialization of the model with static data from the census, surveys etc.

3) Simulation results.

4) Validation of the output results, i.e. comparing the result and the already collected data. This would ensure that model is correct.

The evidence of the better output results by using the real census data can be seen from the Mentat model [38]. The case study of this model revealed the advantages of using the real data for the simulation [39]. Two cases were tested:

1) Running model with random initialization.

2) Running the model with real data initialization.

The comparison of outputs clearly showed the benefits of real data driven simulation. In short, the data driven ABM simulation was closer fit to real data than the randomised simulation.

\section{Towards Full Scale ABM Modelling}

While taking into account the good properties of both the agent based and microsimulation, there is a need to move towards full scale modeling of the population dynamics. There are no general frameworks available which can be used by modelers and government agencies to test the policy before its actual implementation. This full scale model could support all the demography related aspects, such as mortality, health, education, economy, matrimony, fertility, migration, welfare etc.; take into account all of these and simulate the society and public policy. This model would support the micro to macro transition by using actual data and also would be able to analyze useful trends while transition is taking place. The number of agents supported should be large in number (to the tune of millions) and it could make usage of techniques such as distributed computing due to its massive scale

Figure 4 shows the schematic diagram of a module (no. of children a couple would want to have) of such a system. The environment in this diagram would be populated with real data. The rule set would imitate that of the actual world and wherever required, transition probabilities would be used.

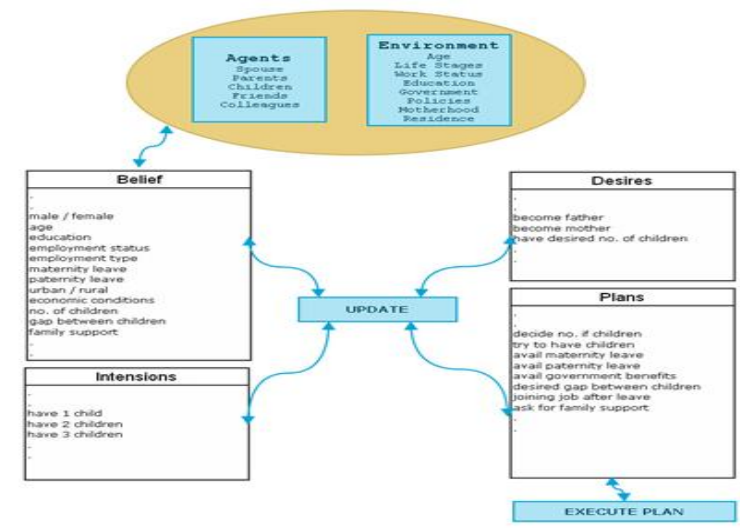

Fig. 4 Proposed Hybrid Model's Module Architecture

\section{Conclusion}

The demographic transitions, population dynamics due to various factors are important topics in the contemporary world society especially when many countries are facing problems like low fertility. There are a number of challenges faced by scientists in study and analyzing these trends, changes, transitions.

Simulation is an important and useful technique which as aided scientists in observing, analyzing these trends as well as to optimize the government policy to maximize the desired effects of various welfare schemes and policies. This paper introduced microsimulation and agent based simulation and stated essential differences in these two techniques. It also analyzed the basic drawbacks of these techniques in context of demographic simulations and population dynamics simulations and suggested the usage of both the techniques simultaneously to achieve micro to macro transition in a full scale general framework, the output of which is close to reality.

\section{Acknowledgment}

This work is supported by the Korea ICT R\&D program of MSIP/IITP (10047117, Development of Distributed/Parallel Multi-Dimensional Demographic Micro Simulation Technologies for Population Dynamics and Socio-Economic Experimentation). The authors also extend their thanks to the whole social simulation team for their constant advice during the development of the work.

\section{References}

[1] M. Myrskyla, Hans-Peter Kohler, and F. Billari, "Advances in development reverse fertility declines." Nature 460 (August 2009): 741743.

[2] M. Caputo, M. Nicotra, and E. Gloria-Bottini, "Fertility Transition: Forecast for Demography." Human Biology (Wayne State University Press) Vol. 80, No. 4 (August 2008): 359-376.

[3] J. Bongaarts, (2001), "Fertility and Reproductive Preference in Posttransitional Societies." Population and Development Review (Population Council) Vol. 27 Supplement: Global Fertility Transition: 260-281.

[4] CIA World Factbook, https://www.cia.gov/ 
[5] Korean Statistical Information Service, http:// http://kosis.kr/eng/

[6] G. H. Orcutt, (1957), "A new type of socio-economic system." The review of economics and statistics: 116-123.

[7] N. Gilbert, K. G. Troitzsch, "Simulation for the Social Scientist" (First Edition, 1999; Second Edition, 2005), Open University Press.

[8] V Neumann, J. and A. Burks, (1966), "Theory of self-reproducing automata." Urbana, IL, University of Illinois Press.

[9] M. Gardner, (1970), "Mathematical games: The fantastic combinations of John Conway's new solitaire game 'Life'." Scientific American 223(4):120-123.

[10] T. Schelling, (1971), "Dynamic Models of Segregation." Journal of Mathematical Sociology 1(2) (1971): 143-186.

[11] M. Wooldridge, N. R. Jennings, (1995), "Intelligent Agents: Theory and Practice", Knowledge Engineering Review, 10(2): 115-152.

[12] S. Franklin, A. Graesser, (1996), "Is it an Agent, or just a Program? A Taxonomy for Autonomous Agents", Proceedings of the Third International Workshop on Agent Theories, Architectures, and Languages, Springer-Verlag.

[13] J. M. Epstein, (1999), "Agent-Based Computational Models and Generative Social Science", Complexity, 4(5): 41-60.

[14] P.M. Torrens, (2004), "Simulating Sprawl: A Dynamic Entity-Based Approach to Modelling North American Suburban Sprawl Using Cellular Automata and Multi-Agent Systems", Ph.D. Thesis, University College London, London.

[15] C. M. Macal, M. J. North, (2005), "Tutorial on Agent-Based Modelling and Simulation", in M. E. Euhl,N. M. Steiger, F. B. Armstrong, J. A. Joines (eds.), Proceedings of the 2005 Winter Simulation Conference.

[16] C. F. Billari, C. Francesco, A. Prskawetz, eds. "Agent-based computational demography: Using simulation to improve our understanding of demographic behaviour." Springer Science \& Business Media, 2003

[17] Todd, M. Peter, C. F. Billari, J. Simao. "Aggregate age-at-marriage patterns from individual mate-search heuristics." Demography 42.3 (2005): 559-574.

[18] C. F. Billari, (2006), "Bridging the gap between micro-demography and macro-demography." In: G. Graziella, J. Vallin and G. Wunsch eds. Demography: analysis and synthesis (4 vols), Academic Press (Elsevier), New York, Vol. 4, pp. 695-707.

[19] C. F. Billari, A. Prskawetz, et al. (2007). —The "Wedding Ring": An Agent-Based Marriage Model Based on Social Interaction.ll Demographic Research, 17: 59.

[20] C. F. Billari, et al. "Agent-based computational modelling: an introduction." Agent-Based Computational Modelling. Physica-Verlag HD, 2006. 1-16.

[21] Hills, Thomas, P. Todd, "Population heterogeneity and individual differences in an assortative agent-based marriage and divorce model (MADAM) using search with relaxing expectations." Journal of Artificial Societies and Social Simulation 11.4 (2008): 5.

[22] A. Diaz, (2010), "Agent Based Models on Social Interaction and Demographic Behaviour", Ph.D. Thesis, Vienna University of Technology, Vienna.

[23] E. Frydenlund, (2011), "Dynamic Attitudes about Motherhood: Modeling Family Planning Policies in Japan”.
[24] E. Silverman, J. Bijak, J. Hilton, V. D. Cao, J. Noble, (2013), "When demography met social simulation: a tale of two modelling approaches." Journal of Artificial Societies and Social Simulation, 16(4), 9.

[25] F. Heiland, (2003). - The collapse of the Berlin wall: Simulating statelevel east to west German migration patternsll. Agent-based computational demography. F. Billari and A. Prskawetz, PhysicaVerlag: 73-96.

[26] Kniveton, Dominic, C. Smith, S. Wood. "Agent-based model simulations of future changes in migration flows for Burkina Faso." Global Environmental Change 21 (2011): S34-S40.

[27] F. Willekens, (2012), "Migration: A perspective from Complexity Science." Paper presented at the Migration workshop of the Complexity Science for the Real World network, Chilworth, UK, 16 February 2012.

[28] Benenson, Itzhak, I. Orner, and E. Hatna, "Agent-based modeling of householders' migration behavior and its consequences." PhysicaVerlag HD, 2003.

[29] N. Geard, J. M. McCaw, A. Dorin, K. B. Korb, J. McVernon, (2013). Synthetic population dynamics: A model of household demography. Journal of Artificial Societies and Social Simulation, 16(1), 8 .

[30] M. Boman, E. Holm (2004), "Multi-Agent Systems, Time Geography, and Microsimulations" ,In Olsson, M. and G. Sjöstedt (Eds.), Systems approaches and their application.

[31] H. Couclelis, (2002), "Modelling Frameworks, Paradigms, and Approaches", in Clarke, K.C., Parks, B.E. and Crane, M.P. (eds.), Geographic Information Systems and Environmental Modelling, Prentice Hall, London.

[32] Mahdavi, Babak, D. O'Sullivan, P. Davis. "An agent-based microsimulation framework for investigating residential segregation using census data." (2007): 365-371.

[33] R. Boero, F. Squazzoni, "Does empirical embeddedness matter? Methodological issues on agent-based models for analytical social science." Journal of Artificial Societies and Social Simulation 11 (2005).

[34] J. S. Dean, G. J. Gumerman, J. M. Epstein, R. L. Axtell,A. C. Swedlund, M. T. Parker, S. McCarroll, In: Understanding Anasazi culture change through agent based modeling. Oxford University Press (2000) 179-205.

[35] J. M. Galan, A. Lopez-Paredes, O. del Olmo, "Effect of technological diffusion of water conservation measures in an ABM-GIS integrated model." In: VI International Workshop on Practical Applications of Agents and Multiagent Systems, Salamanca: Universidad de Salamanca (2007) 169-180.

[36] A.L. Barabasi, H. Jeong, Z. Neda, E. Ravasz, A. Schubert, T .Vicsek, "Evolution of the social network of Scientific collaborations." APS Meeting Abstracts (March 2002) 27012.

[37] D. Gatherer, "Comparison of Eurovision song contest simulation with actual results reveals shifting patterns of collusive voting alliances." Journal of Artificial Societies and Social Simulation 11 (2006).

[38] S. Hassan, J. Pavon, M. Arroyo, C. Leon, "Agent based simulation framework for quantitative and qualitative social research: Statistics and natural language generation." In Amblard, F., ed.: ESSA'07: Fourth Conference of the European Social Simulation Association, Toulouse, France (2007) 697-707.

[39] G.N. Gilbert, S. Hassan, J. Pavon, (2008), "Injecting data into simulation. Can agent-based modelling learn from Microsimulation." 